\title{
The Salt-Free Dyeing on Cotton: An Approach to Effluent Free Mechanism; Can Chitosan be a Potential Option?
}

\author{
Kunal Singha ${ }^{1, *}$, Subhankar Maity ${ }^{1}$, Mrinal Singha $^{2}$ \\ ${ }^{1}$ Department of Textile Technology, Panipat Institute of Engineering \& Technology, Haray ana, India \\ ${ }^{2}$ Department of Pharmaceutical Chemistry, CU Shah College of Pharmacy \& Research, Gujarat, India
}

\begin{abstract}
The present study utilizes salt free dyeing on cotton which would not con tribute to environmental pollution an undertaken to explore promising approach to reduce cost of dye process. The salt free dyeing used to dye cotton. In conventional Method of dyeing of cotton with reactive dyes, alkali $\mathrm{P}^{\mathrm{H}}$ is should maintain in the dye bath. This method requires more electrolytes for exhaustion and alkali for fixation. In this paper the fibre modification technique based on polyacrylamide was discussed. When the fabric is treated with polyacryla mide (chitosan), the primary hydroxyl groups of cellu lose is (partially) modified into a mide groups, which intern leads the cellulose to act like as wool fibre and hence rea ctive dyes can be dyed on cotton at neutral $\mathrm{P}^{\mathrm{H}}$ in the absence of electrolyte and alkali.
\end{abstract}

Keywords Salt Free Dyeing, Reactive Dye, PH, Polyacrylamide (Chitosan), Cellulose, A mide Groups

\section{Introduction}

Cotton fibres are widely applied in textile industry due to its excellent properties of hygroscopicity, air permeability, biodegradability, no static electricity, etc. The dyeing of these fibres are generally done with reactive dyes due to its brilliancy, variety of hue, high wet fastness, convenient usage and high applicability. These reactive dyes contain a reactive group, either a haloheterocycle or an activated double bond, that, when applied to a fibre in an alkaline dye bath, forms a chemical bond with hydroxyl group on the cellu losic fibre [1]. In recent years, reactive dyes maintain the largest annual consumption in the world among the dyes used for which establishes its important status in the dye manufacture industry. But some problems, such as low dye utilization, large a mount of electrolyte used and high volume of wastewater discharged, always exist in the application of reactive dyes. The dyeing of one kilogram of cotton with reactive dyes demands from 70 to 150 litre water, $0.6-0.8 \mathrm{Kg}$ $\mathrm{NaC} 1$ and from 30 to $60 \mathrm{~g}$ dyestuffs [2]. Due to these problems this class of dyes is the most unfavorable one from the ecological point of view, these effluents produced gives high values of BOD/COD (Biological oxygen demand / Chemical oxygen demand) and increases salinity of the rivers affects the delicate biochemistry of aquatic life. More than 80,000 tons of reactive dyes are produced and consumed each year, making it possible to quantify the total amount of pollution caused by their use. So, most researchers

* Corresponding author:

kunalsingha28@gmail.com (Kunal Singha)

Published online at http://journal.sapub.org/textile

Copyright (C) 2012 Scientific \& Academic Publishing. All Rights Reserved focus on introducing Salt-free/low-salt dyeing technology for reactive dyes.

\subsection{Advantages of the Salt-Free Cotton Dyeing over the Conventional Way}

Chitosan will be used for modification of cotton fabric and then fabric will be dyed with conventional dyeing procedure without use of salt[2,3]. There are certain advantages in salt free dyeing over the conventional dyeing technique, for example;

- Elimination of salts an electrolyte.

- Maximum fixation of dyes.

- Minimu m hydrolysis of dyes.

- Low volu me of water requirement during the wash off process.

- To increase the reactivity of reactive dyes.

- To increase the wash fastness and rubbing fastness of pretreated sample.

- To increase the fabric crease recovery and flexural rig idity environ mentally friendly.

- Significant savings in process costs.

\subsection{Techni ques Used for Salt-Free Dyeing Process}

The following way are generally used to achieve the salt-free dyeing over cotton fabric using reactive dyes by modifying the cotton surface after introducing some new functional group with having reactive dye-affin ity.

\section{Modification of Reactive Dyes}

It is reported that by using reactive dyes containing two or more reactive groups rather than one reactive group per dye 
molecule fixation can be increase from $60 \%$ to $80 \%$. In practice this method can be productive owing to the detrimental effect that additional group can have on migration, leading to lower fixation, especially at higher depths. Most suitable of this type of dye is Cibacron LS range. Two hetrobifunctional groups mixed to form multifunctional reactive dye. It utilizes several different types of reactive groups. Monoflourotriazine and monochlorotriazine in combination with sulphone can be used. Due to higher molecular weight of this dye it function effectively In presence of reduce amount of salt but its mo lecular weight is high so migration capacity is low. So it is applied in presence of small amount of electrolyte for only 30 minutes at neutral condition for $90^{\circ} \mathrm{C}$ followed by addition of alkali to effect fixation, generally over 60 minutes but at $70^{\circ} \mathrm{C}$ to promote fixation. Migration and leveling occur in higher temperature but the fixation occurs slowly at lower temperature. In present days novel bis-sulphatoethyl sulphone are vailable in the market which consists of two ureido groups. Build up well in absence of electrolyte, here ureido group help to increase substantively leading to lower use of salts $[3,4]$.

\subsection{Advantages}

Low a mount of salt required.
Dyeing time is less.

Less effluent problem

Eco-friendly in nature

\section{Modification of cellulose or Cationization of cotton: Using Chitosan (polyacrylamide)}

In this process a new fiber modification technique based on cationic acrylic copoly mer is retreated with cotton fiber because it believed that pre-treated of cellulosic fiber with Polymer to offer an opportunity for increasing both the substantivity and reactivity of fibers towards reactive dyes under neutral conditions. The nature of a reactive poly mer resin is such that it may react with nucleophilic sites in cellulosic fibers or in the polymer itself, thus fixing the polymer to the substrate. During subsequent dyeing, further reactions between the polymer and the dyestuff, the fiber and the dyestuff, and the fibre and the polymer and can be expected to take place, forming cross-lin $\mathrm{k}$ within the fibers.

\subsection{Processing of Chitosan}

The preparation of chitos an is very easy as shown by fig 1 and fig 2 .

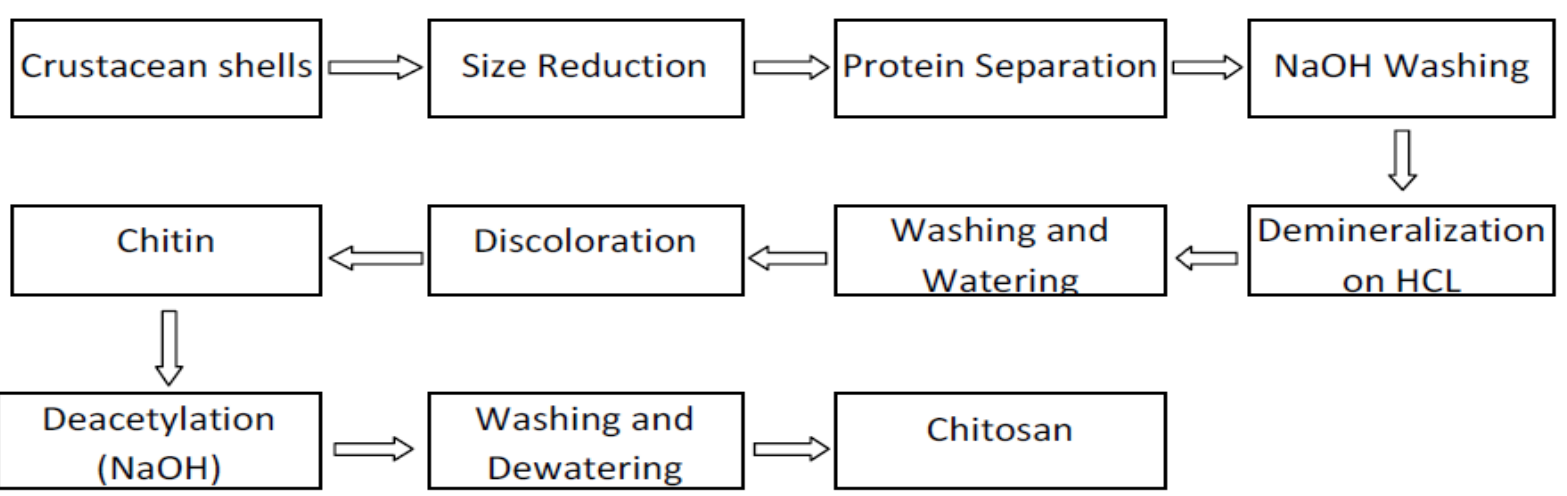

Figure 1. Steps invoved in the processing of chitosan[2,14] 


\section{Chemical Structure}

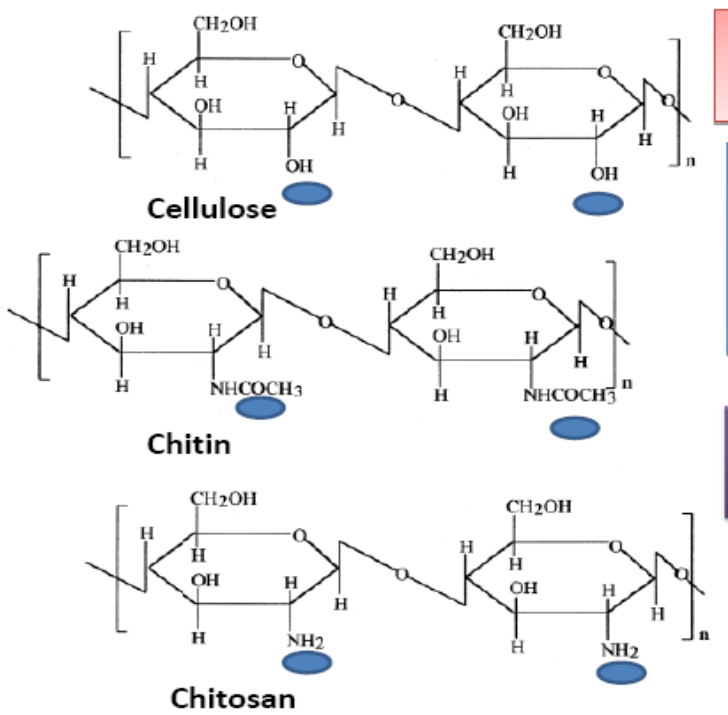

Cellulose (1-4)-2-deoxy-glucopyranose units

Chitin regarded as cellulose with hydroxyl at position C2 replaced by an acetamido group.

chitin is poly $(-(1-4)-\mathrm{N}-$ acetyl-d-glucosamine)

\section{Properties of Chitosan}

- Linear polyamine

- Reactive A mino Groups

- Reactive Hydroxyl Groups are available

- Water soluble and positively charge at acidic $\mathrm{P}^{\mathrm{H}}$

\subsection{Principle Involved In Chitosan Dyeing Process on Cotton Fabric}

The pretreated cotton with polymer is generally used for the chitosan dyeing process for a cotton fabric. The pretreatment of cotton fabric with polyacrylamide demonstrates the introduction of functional amino groups which increase the substantivity and also the reactivity of cotton. The cationic charged amino groups may be involved in the adsorption of anionic chromophore of reactive dyes. The improved dye ability is postulated due to the presence of amide groups $\left(-\mathrm{CONH}_{2}\right)$ available from the polyacrylamide which also tents to improve the reactivity of cellulosic substrate. The attachment of the dye molecules onto the partially-modified cellulosic substrate is by covalent bonding since no dyes strips out from the dyed sample (fig. 3). This is also indicative through the fastness properties wash fastness. The fastness values of all such dyed samples are quite satisfactory and comparable with those of conventional dyed samples. The dry crease recovery angle values of the polymer treated samples are $80^{\circ}$ while that of conventional dyed sample is $68^{\circ}$. Therefore, as expected, the polymer treated dyed samples indicate an improvement in the wrinkle recovery.

A high level of dye exhaustion on the treated fabric can be achieve in the absence of salt and alkali at a temperature as low as (Normally at $60-80^{\circ} \mathrm{C}$ ) that used in the conventional dyeing process. Further increases in temperature may improve dye bath exhaustion, but only to a limited extent. However higher temperature $\left(90-100{ }^{\circ} \mathrm{C}\right.$ ) are generally recommended for dyeing modified fabrics to obtain better penetration and fixation of dye $[5,6]$.

\subsection{Advantages}

Pretreatment of cotton with polyacrylamide enhances the possibility of dyeing cotton at neutral $\mathrm{pH}$.

- Such pretreatment, as applied through pad - dry - cure process, brings about some chemical changes in the treated fabric.

- Fastness properties are adequate and quite comparable with conventionally dyed samples.

- The wrinkle resistance of the dyed fabric also improves.

- The dyeing of cotton with reactive dyes using polyacrylamide in the dye bath improves the dye ability of cellulosic fabrics with reactive dyes and reducing effluent discharge[7].

\subsection{Using CHPTAC}

Che mically cationized cotton is usually produced by the etherifying reaction of cotton with the tertiary amino or quaternary ammonium cationizing reagents, especially quaternary ammonium cationizing reagents (fig. 4), such as 2,3-epoxypropyltrimehtyla mmonium chloride. This compound is usually formed in situ from the reaction of sodium hydro xide with 3-chloro-2-hydro xypropyltrimethyla mmoniu m chloride (CHPTAC)[12]. 
Structure of

polyacrylamide

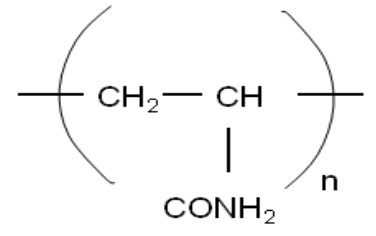

Reaction

Cellulose polyacrylamide
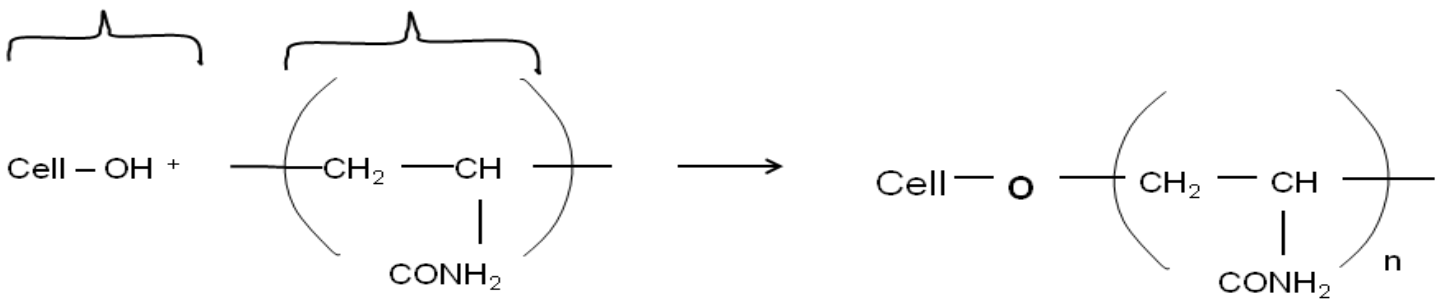

Figure 3. Reaction of cellulose with poly acrylamide
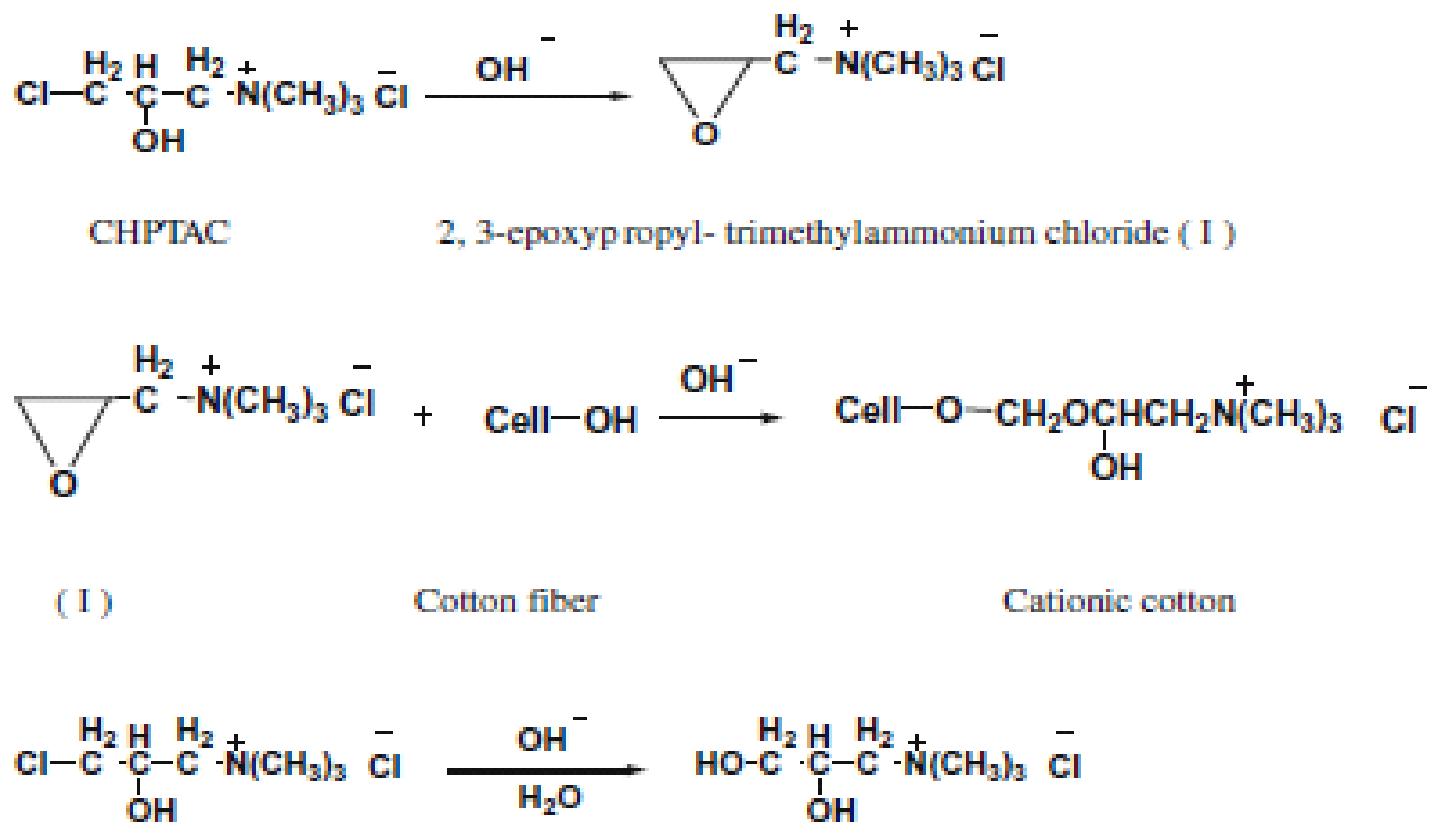

Figure 4. Reactions occurred during the cationization process of cotton 


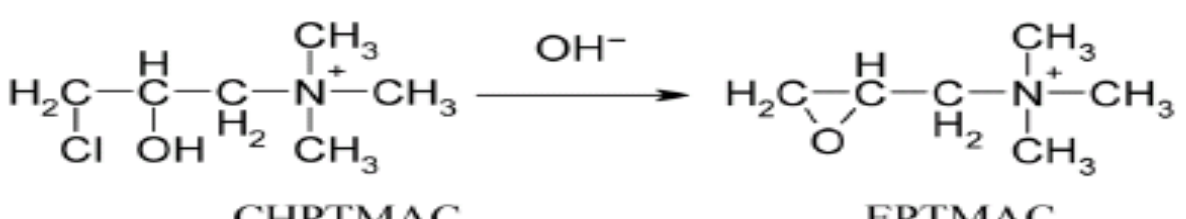

Scheme 1. Reaction of CHPTMAC with alkali.<smiles>[R]CC(O)C(C[N+](C)(C)C)O[R]</smiles>

Scheme 2. Reaction of EPTMAC with alcohol.

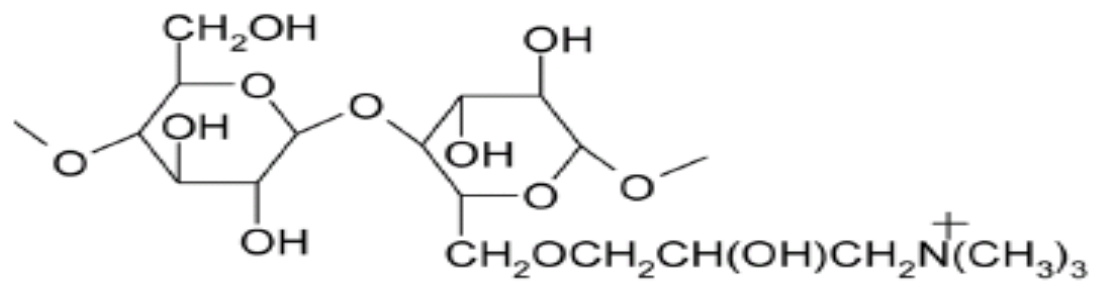

Seheme 3. Cationized cotton.

Figure 5. Chemical structure of CHPTMAC and its cross-linking react ion with cotton

\subsection{Pre-treatment of Cotton with CHPTMAC}

The solution consisting of $35 \mathrm{~g} / 1$ of 3-chloro-2-hydroxy propyl trimethyl ammonium chloride (CHPTMAC) $(65 \%$ $\mathrm{w} / \mathrm{w}$ ) and $15 \mathrm{~g} / \mathrm{l}$ of sodium hydroxide was applied to the well-p repared cotton fabric. The wet pick up was $100 \%$ and to minimize the hydrolysis of reactant, the alkali was added to the bath just before application. The fabric was stored at room temperature for $24 \mathrm{hr}$, and then rinsed several times with water and finally neutralized with a dilute acetic acid (1g/1). Reaction of CHPTMAC with Cellulose 2,3epoxypropyltrimethylammonium chloride (CHPTMAC) was prepared in situ by the reaction of 3-chloro-2-hydroxypropyltrimethylammonium chloride (EPTMAC) with alkali as shown in Scheme 1. EPTMAC will react with alcohols under alkaline conditions to form ethers (step 2) and will thus produce a modified fiber when it reacts with cotton as shown in step 3 . As a result, cotton will have cationic dye sites covalently bound to the polymer chains. These dye sites will strongly attract anionic reactive dyes, enabling the use of these dyes without the large a mount of electrolyte (fig. 5).

\section{Dyeing of Fabric with CHPTMAC}

Dyeing of pre-treated cotton was carried out in the absence of salt by the conventional method. The results showed that all of the dyes gave an excellent exhaustion with $1 \%$ o.w.f. and most of them showed a high fixation with good levelness exhaustion, fixation and color strength values for the most dyes on the treated cotton is higher than those on the untreated cotton. The fixation values varied from dye to dyes. In these dyeing systems, the strong attraction between the cationic dye sites on the modified cotton and the anionic dyes existed which led to obtain a very high exhaustion rates without addition of electrolytes to the dye-bath. The color strength of most of the dyeing on the treated cotton was often twice and in some cases up to four times of untreated cotton[8,9]. However, these high color strength values might be partially attributed to surface dyeing or ring dyeing. The wash fastness of the cationized dyed cotton was similar to that of the untreated dyed fabrics and the cross staining results were good The excellent light fastness of the reported dyeing on cationic cotton is particularly striking, since cationic pretreatments, as a general rule, are known to impair light fastness. The cationic pretreatment used here is nonpolymeric in nature and can be expected to penetrate the cotton fiber prior to fixation. The rubbing fastness of both sets of samples was good. The observation of the dyed samples also showed a good level of uniformity. An enhancement of the color strength is expected when the dye concentration is increased, since a greater number of dye molecules would be available in the vicinity of the cotton cellu lose at higher concentrations. Unlike untreated cotton, however, the dye build-up on the treated cotton is limited by its saturation adsorption value which is related to the amount of EPTMAC applied during pretreatment. It can be seen from Figure 1 that the cotton treated with EPTMAC obtained good color strength (fig. 6) at all depth of shades below $4.5 \%$ o.w.f. Above this level, there is no increase in color 
strength with increasing the dye concentration. However, the color strength on the treated fiber dyed with $5 \%$ o.w.f. dye is similar to that of the color strength of an $8 \%$ o.w.f. conventional dyeing.

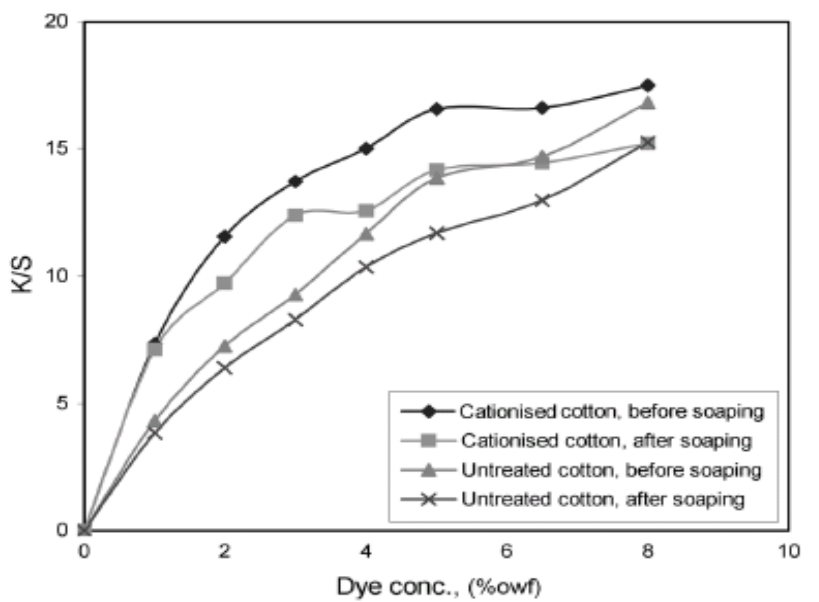

Figure 6. Build-up properties of Cibacron Red HF for treated and untreated cott on fabric influence of Electrolyte in Dyebath

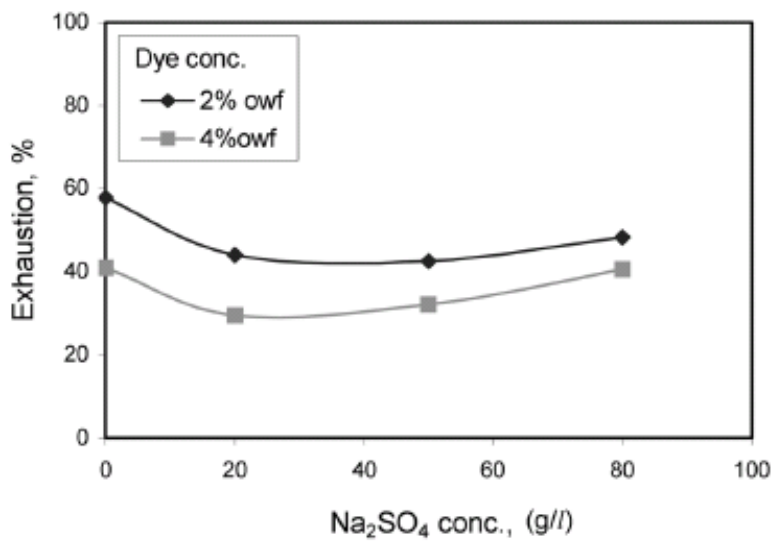

Figure 7. Effect of electrolyte concentration on exhaustion of Cibacron Red HF for treated cotton fabrics

The effect of salt on the dyeing of the quarternized cotton is shown in Figure 4. In the dye concentrations applied to the fabrics well below the saturation limit of the treated fibers, the degree of exhaustion decreased with increasing salt concentration[10] and for the $2 \%$ o.w.f. Cibacron Red HF, the treated cotton gave $60 \%$ exhaustion of anionic dye in the absence of electrolyte, but the exhaustion droped to $45 \%$ after adding $20 \mathrm{~g} / \mathrm{l}$ of sodium sulphate. This unfavorable effect was because of the sulphate anions which have a significant affinity for qurternized cellu lose and they were able to compete with dye anions for the quaternary sites in the modified fibers with increase in salt concentration [11], as nearly all the quaternary sites are occupied and the fiber became neutrally or even negatively charged (fig.7). In this way dye adsorption was the same as the untreated cotton.

\section{Grafting of Cellulosic Material: Using MAPTAC}

Graft polymerization of the cationic monomer methacrylol amino propyltrimethyl ammonium chloride (MAPTAC), on to scoured cellulose onto scoured cellulose was carried out in bleaching process, aiming to modify the fibre using a single bath. The extent of MAPTAC fixation on cellulose was measured .The bleaching performance of hydrogen peroxide in presence of the modifying agent was found to slightly reduce. The modified bleached cotton fabric was then dyed with a commercial reactive dye in the absence of salt. The dye uptake and colour strength of the modified fabric was markedly increased with an increase in concentration of MAPTAC. This was attributed to the presence of cationic group of MAPTAC which play a crucial role in attracting the anionic dyes from the dye bath[12]. Cationic monomer which contains C-C double bond can be grafted using redox initiator. Fixation of MAPTAC achieved by graft polymerization using potassium per sulphate as redox initiator, it oxidizes the cellulose hydroxyl group, produce cellulose hydroxyl free radical, which initiate the graft polymerization, the enhanced dye ability of the modified cotton fabrics were attributed to the presence of cationic groups which have large affin ity for anionic dyes.
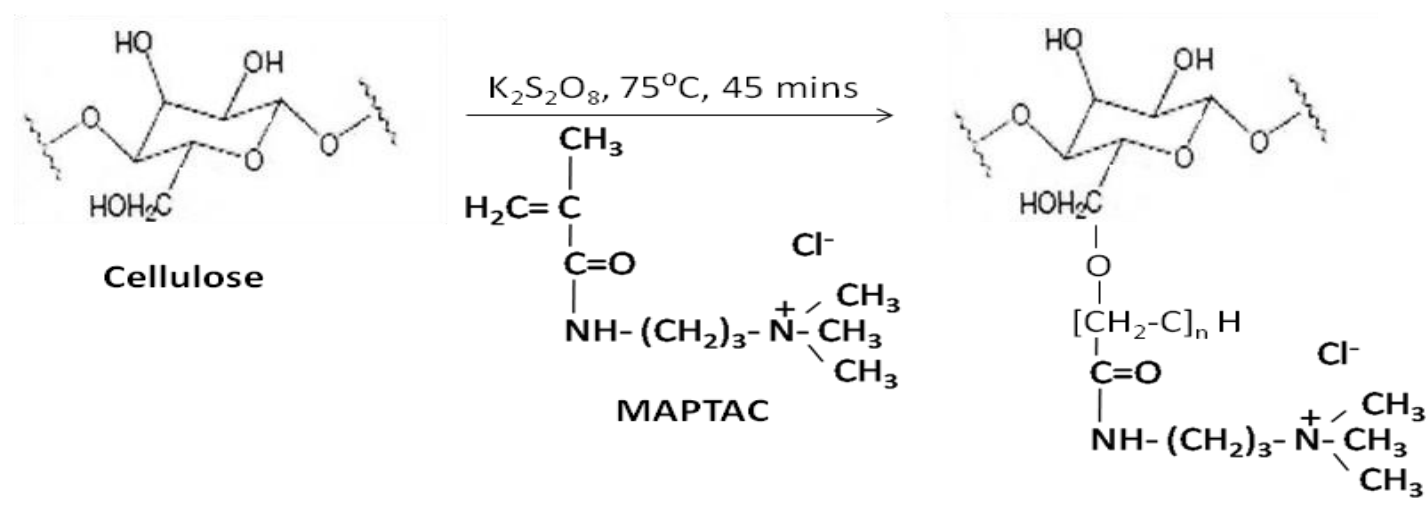

Cellulose cross-linked product with MAPTAC

Figure 8. Pretreatment of Cotton Fabrics for Grafting 
Firstly cotton fabric is treated with different concentrations of MAPTAC ranging from $0-50 \mathrm{~g} / 1$ in presence of potassium persulphate as redox initiator (fig. 8). The treatment occurs at $75^{\circ} \mathrm{C}$ and MLR is $1: 20$ for $45 \mathrm{mins}$, $5 \mathrm{~g} / 1$ hydrogen peroxide, $2 \mathrm{~g} / 1 \mathrm{NAOH}, 1 \mathrm{~g} / 1$ sodium silicate added and the temperature raised to $95^{\circ} \mathrm{C}$. This temperature is maintained for $30 \mathrm{~min}$ before fabric is removed from the solution[13].

\subsection{Disadvantages of this Process}

\section{Colour Fastness to Light}

Light fastness decreases with an increase in the amount of modifying agent, cationic group may act as photocatalyst because of lack of deactivating nitro group on the dye chromophore combine with prevention of dye aggregation by the presence of cationic groups, leading to acceleration of chromophore decomposition under the light[13].

Ring Dyeing

Since the compound carries the cationic group, the substantivity towards cotton fiber should be high enough to force the modifying agent to diffuse into inner portion of the fiber. By graft copolymerization we get high molecular weight polymer. If the grafted compound will be high molecular weight its mig ration will be low but substantivity is high as results such compound remain on yarn surface and lead to ring dyeing.

- Using dendrimers on cotton fabric surface

In contrast to linear polymers, dendrimers are highly branched, fractal-like macromolecules of well-defined, three-dimensional structure, shape and topology. From their shapes are derived names such as arborols, cascade, or star-burst polymers. The synthes is typically proceeds in stages or generations in which both the extent of branching and the size of the dendrimer increase; synthesis can be closely controlled to achieve a given size, shape and internal structure. Dendrimers cannot engage in chain entanglements as do linear poly mers and thus are not useful in mechanical applications as are conventional linear poly mers; generally, dendrimers are viscous liquids at room temperature. Functional end groups impart to the dendrimer characteristic physical and chemical properties, such as solubility, compatibility with plastics, uptake of guest molecules and surface activity $[10,11]$.

\section{Application of Dendrimer}

Samples of cotton were treated with the den-drimer (fig. 9) at $90^{\circ} \mathrm{C}$ at three different $\mathrm{pH}$ values $(4,7$ and 9) and at four different concentrations $(0.25,0.5,1.0$ and $1.5 \%$ o.w.f.), using a 15:1 liquor ratio[11].
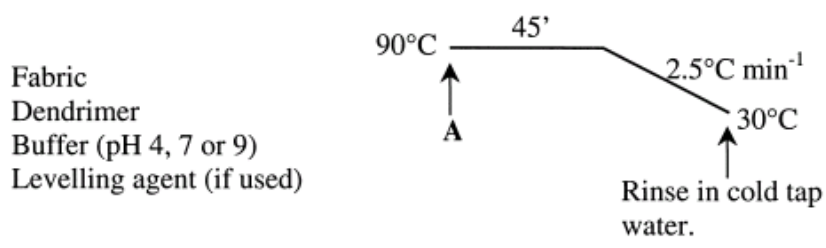

Figure 9. Application of dendrimer on fabric

\subsection{Dyeing}

To highlight differences in dye uptake between the pre-treated and untreated cotton samples, the majority of dyeing were carried out using a competitive dyeing method (Fig. 8) in which a sample of dendrimer-treated cotton and a sample of untreated cotton were dyed competitively in the same dye bath. All dyeing were carried out using a liquor ratio of 20:1. After dyeing, the samples were washed-off[10]. In addition, a modified method of dye application was used to investigate the application of dye without salt and/or alkali. Accordingly, these modified dyeing processes were carried out both unbuffered as well as at a buffered $\mathrm{pH}$ of 4.0 and 7.4.

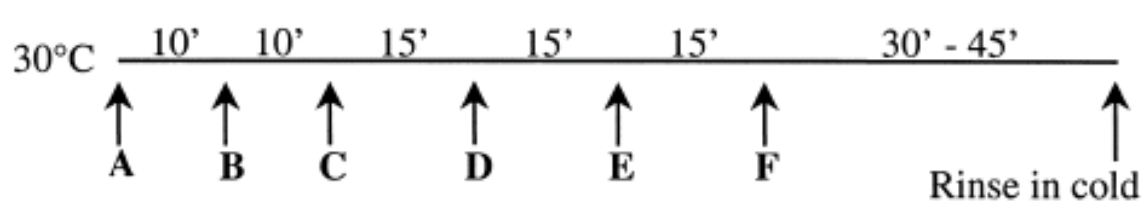

$\begin{array}{ll}\text { A } & \text { Dye \& Fabric } \\ \text { B } & \mathrm{NaCl} \\ \text { C } & \mathrm{NaCl} \\ \text { D } & \mathrm{NaCl} \\ \text { E } & \mathrm{Na}_{2} \mathrm{CO}_{3} \\ \text { F } & \mathrm{Na}_{2} \mathrm{CO}_{3}\end{array}$

$$
\begin{aligned}
& 2.5 \mathrm{~g} \mathrm{dm}^{-3} \text {. } \\
& 7.5 \mathrm{~g} \mathrm{dm}^{-3} \text {. } \\
& \text { Remaining amount } \\
& 10 \% \text { of the required amount } \\
& \text { Remaining amount }
\end{aligned}
$$

Rinse in cold tap water till clear water is detected.

Figure 10. Schematic process of the dyeing method 


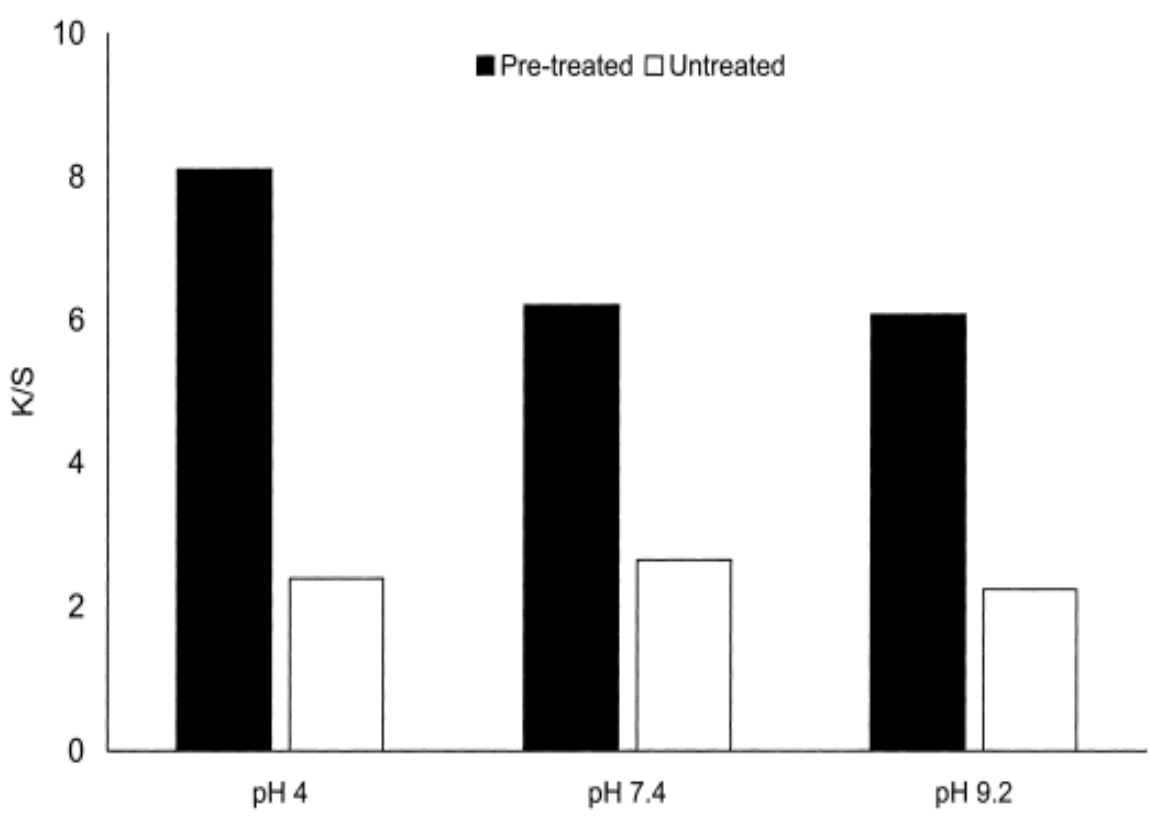

Figure 11. Dependence of color strength on pretreat ment on cotton with dendrimers



Figure 12. Effect of pretreatment with varying amounts of dendrimer on color strength

It shows that for each of the three $\mathrm{pH}$ values used for dendrimer application, the color strength of the pretreated cotton was considerably higher than that of the corresponding untreated cotton (fig. 10).

The effect of pretreatment with varying amounts of dendrimer on color strength is shown in Fig. 11 and generally, color strength increased with increasing a mount of dendrimer applied, although the observed increase (fig. 12) in color strength was small[10].

\subsection{Use of Organic Salt: Sodium Edate (SE)}

Sodiu medate was used in the dyeing of cotton fabrics with reactive dyes. Exhaustion behaviour of this organic electrolyte was compared with sodium sulphate. Also, using this electrolyte for reactive dye fixation was evaluated in comparison with sodium carbonate[14].

\subsection{Dyeing Conditions}

In a dyebath containing different amounts of SE (50 g/l) and $2 \%$ shade of CI Reactive Blue 19 or CI Reactive Black 5 with liquor ratio $40: 1$, cotton fabric was added at $35^{\circ} \mathrm{C}$ and the temperature was raised to $60^{\circ} \mathrm{C}$ or $80^{\circ} \mathrm{C}$ over $20 \mathrm{~min}$ for vinyl sulphone or monochlorotriazine dyes, respectively. After which time the dyeing was continued at $60^{\circ} \mathrm{C}$ for 30 min (mig ration phase). Then $20 \mathrm{~g} / \mathrm{l}$ of sodium carbonate was added portion wise and the dyeing was maintained at $60^{\circ} \mathrm{C}$ for further $45 \min [15]$. 


\section{Conclusions}

The purpose of this paper is to give a overall idea about the pre-treatment of cotton with a chitosan could enhance the dye ability of the fibre with reactive dyes. The chitosan contains primary amino groups, with which theoretically, a reactive dye should be able to react under neutral/acidic $\mathrm{P}^{\mathrm{H}}$ conditions [16]. It is also decided to examine whether or not the chitos an could, under appropriate $\mathrm{P}^{\mathrm{H}}$ conditions, as sume a positive charge and so permit "salt-free" dyeing.

Pretreatment of cotton with chitosan enhances the possibility of dyeing cotton at neutral $\mathrm{P}^{\mathrm{H}}$ with various commercial reactive dyes and such pretreatment also brings about some chemical changes in the treated fabric. Fastness properties are adequate and quite comparable with conventionally dyed samples. The bending resistance of the dyed fabric also improves. The dyeing of cotton with reactive dyes using chitos an with cross-linker in the dye bath improves the dye ability of cellulosic fabrics with reactive dye[17], when dyeing the modified substrates; reactive dyes can be much more efficiently exhausted and fixed onto cellu losic fabrics under neutral conditions in the absence of salt and alkali. The modifications show an overall suitability for different reactive dyes. The modified dyeing do not suffer either from a significant drop in colour strength, wash fastness or from duller shades.

\section{Abbreviations}

BOD: Biological oxygen demand; COD: Chemical oxygen demand; CHPTMAC: 3-chlo ro-2-hydro xypropyltri methylammon iu m chloride; g/l: gram per litre; w/w: weight by weight ratio; EPTMAC: 3-chloro-2-hydro xypropyltrimet hylammonium chloride; o.w.f.: on the weight of the fabric; conc.: concentration; K/S: Kubelka-Munk color value, SE: Sodiu m Edate.

\section{REFERENCES}

[1] I D Rattee: Reactive dyes for cellu lose, Coloration 14 (1984) 1953- 1983.

[2] C Allegre: Treatment and reuse of reactive dy eing effluents, Journal of Membrane Science 269 (2006) 15-34.
[3] Philippe Moulin: Savings and re-use of salts and water present in dye house effluents, Desalination 162 (2004)13-22.

[4] J A Tay lor: Recent developments in reactive dyes, Coloration 30 (2000) 93-107.

[5] A H M Renfrew: Cellulose reactive dyes: recent developments and trends, Coloration 20 (1990) 1-10.

[6] F Lehr: Synthesis and Application of Reactive Dyes with Heterocy clic Reactive Sy stems, Dyes and Pigments 14 (1990) 239-263.

[7] Lili Wang, Wei Ma: Preparation of cationic cotton with two-bath pad-bake process and its application in salt-free dyeing, Carbohy drate Poly mers 78 (2009) 602-608.

[8] S M Burkinshaw: The use of dendrimers to modify the dy eing behaviour of reactive dyes on cotton, Dyes and Pigments 47 (2000) 259-267.

[9] E Peter: Dendrimers and dye - a review, Dy es and Pigments 48 (2001) 187-195.

[10] S M Burkinshaw, Lei, D M Lewis: Modification of cotton to improve its dy eability, 3 (2006) 344-376.

[11] S D Bhattacharaya, B J Aggarwal: A novel of technique of cotton dy eing at reactive and at neutral PH (2009) 778-807.

[12] Salt-free dyeing- A new method of dyeing on lyocell/cotton blended fabrics with reactive dyes, Aravin Prince Periy asamy, Bhaarathi Dhurai, K.Thangamani, AUTEX Research Journal, Vol. 11, No1, March 2011.

[13] Rosemarie, T. September 22, 1987, Patent:USP 4,695,289, Process for improving the color yield and wet fastness properties of dyeing or prints produced on cellulosic fabrics with anionic dyes: treatment with cationic imidazole.

[14] $\mathrm{Bi}$-and poly-functional reactive dyestuffs, their preparation and their use United States Patent 5597902.

[15] Compounds useful for the preparation of bi-and polyfunctional reactive dyestuffs United States Patent 5686642 .

[16] A.Georgieva, D.Pishev. Dyeing Of Cellulose Textile Materials With Mono - And Polyfunctional Reactive Dyes. Journal of the University of Chemical Technology and Metallurgy, XXXVI, Book 2, 2001- Sofia, Bulgaria.

[17] J. Richard Aspland. Textile dyeing and coloration. American Association of Textile Chemists and Colorists.

[18] Feng Zhang Yuyue Chen, Hong Lin Hao, Wang and Bing Zhao: $\mathrm{HBP}-\mathrm{NH}_{2}$ grafted cotton fiber: Preparation and salt-free dy eing properties, Carbohy drate Poly mers 74 (2008) 250-256. 